PROCEEDINGS OF THE

AMERICAN MATHEMATICAL SOCIETY

Volume 128, Number 2, Pages 503-510

$\mathrm{S}$ 0002-9939(99)04990-4

Article electronically published on June 21, 1999

\title{
SPECTRAL TYPES OF SKEWED BERNOULLI SHIFT
}

\author{
YOUNGHO AHN AND GEON HO CHOE
}

(Communicated by Theodore W. Gamelin)

\begin{abstract}
For the transformation $T: x \mapsto k x(\bmod 1)$ for $k \geq 2$, it is proved that a real-valued function $f(x)$ of modulus 1 is not a multiplicative coboundary if the discontinuities $0<x_{1}<\cdots<x_{n} \leq 1$ of $f(x)$ are $k$-adic points and $x_{1} \geq \frac{1}{k}$. It is also proved that the weakly mixing skew product transformations arising from Bernoulli shifts have Lebesgue spectrum.
\end{abstract}

\section{INTRODUCTION}

Given a measure-preserving transformation $T$ on a probability space $X$ and a measurable function $\phi: X \rightarrow \mathbb{T}=\{z \in \mathbb{C}:|z|=1\}$, we define an isometry $V^{\phi, T}$ on $L^{2}(X)$ by $\left(V^{\phi, T} f\right)(x)=\phi(x) f(T x)$. If $T$ is invertible, then $V^{\phi, T}$ is unitary. When $T$ is an irrational rotation, the properties of $V^{\phi, T}$ are investigated in [4], [5], [8], [10]. In [5] it is proved that, if $\phi$ is a step function with its discontinuities at rational points, $V^{\phi, T}$ has no eigenfunction. In this paper, we are interested in the case when $T$ is a Bernoulli shift. We also investigate the skew product transformation associated with $\phi$ defined on $X \times \mathbb{T}$ by $T_{\phi}(x, z)=(T x, \phi(x) z)$. For the spectral properties of $T_{\phi}$, see [2], [7].

A function $\phi$ of modulus 1 is called a (multiplicative) coboundary if there exists $q(x)$ such that $\phi(x)=\overline{q(x)} q(T x),|q|=1$ a.e. on $X$. Let $\mathcal{M}=\left\{h \in L^{2}(X)\right.$ : $U h=h\}$. If $T$ is ergodic and $\phi$ is real-valued, i.e. $\phi(x)=\exp \left(\pi i \chi_{E}(x)\right)$ where $\chi_{E}$ is a characteristic function of $E$, then the dimension of $\mathcal{M}$ is 0 or 1 . If $\operatorname{dim}$ $\mathcal{M}=1$, then (i) $\phi(x)$ is a coboundary, and (ii) there exists $q$ such that $q(x)=$ $\exp \left(\pi i \chi_{F}(x)\right)$ for some $F$, $\exp \left(\pi i \chi_{E}(x)\right)=q(x) q(T x), E=F \Delta T^{-1} F=F^{c} \Delta$ $T^{-1} F^{c}$. Throughout the paper all the set equalities, set inclusions and function equalities are understood as being modulo measure zero sets and all the subsets are measurable unless otherwise stated.

Numbers of the form $\sum_{i=1}^{n} a_{i} k^{-i}, a_{i} \in\{0, \ldots, k-1\}$, are called $k$-adic numbers and denoted by $\left[a_{1}, a_{2}, \ldots, a_{n}\right]$. For $k=2$ they are called dyadic numbers. We will regard 1 as a $k$-adic number. In [1] it is shown that if $0 \leq a<b \leq 1$ are dyadic numbers and if $\exp \left(\pi i \chi_{[a, b]}\right)$ is a coboundary for $T: x \mapsto 2 x(\bmod 1)$, then $a=\frac{1}{4}, b=\frac{3}{4}$.

Received by the editors July 25, 1997 and, in revised form, March 31, 1998.

1991 Mathematics Subject Classification. Primary 28D05, 47A35.

Key words and phrases. Coboundary, metric density, weakly mixing, Lebesgue spectrum, Bernoulli shift.

The second author's research was supported by GARC-SRC and KOSEF 95-07-01-02-01-3.

(c)1999 American Mathematical Society 
Let $F$ be a Lebesgue measurable subset of $\mathbb{R}$ and $\lambda$ the Lebesgue measure on $\mathbb{R}$. For a point $x \in \mathbb{R}$ the metric density of $F$ at $x$ is defined to be

$$
d_{F}(x) \equiv \lim _{r \rightarrow 0+} \frac{\lambda(F \cap(x-r, x+r))}{2 r},
$$

provided that this limit exists. The metric density of $F$ equals 1 and 0 at a.e. point of $F$ and $F^{c}$, respectively. If $(x-r, x+r)$ and $2 r$ are replaced by $[x, x+r)$ and $r$, respectively, in the above limit, then we call the corresponding limit $d_{F}^{+}(x)$ the right metric density of $F$ at $x$. Similarly the left metric density $d_{F}^{-}(x)$ is defined. Recall that for $f \in L^{1}(\mathbb{R}), x \in \mathbb{R}$ is called a Lebesgue point of $f$ if

$$
\lim _{r \rightarrow 0+} \frac{1}{2 r} \int_{(x-r, x+r)}|f(y)-f(x)| d \lambda(y)=0 .
$$

It is known that for $f \in L^{1}(\mathbb{R})$ almost every $x$ is a Lebesgue point of $f$. If $x$ is a Lebesgue point of $\chi_{F}$, then $d_{F}(x)=d_{F}^{+}(x)$ (see [13]). The metric density of $F$ at a specific point may not be defined, hence it is not a Lebesgue point of $\chi_{F}$ : For $\kappa$ and $\eta, 0 \leq \kappa \leq \eta \leq 1$, there exists $F \subset \mathbb{R}$ so that the upper and lower limits of $\lambda(F \cap(-\delta, \delta)) / 2 \delta$ are $\eta$ and $\kappa$, respectively, as $\delta \rightarrow 0$ (see [9]). Recall that for a point $x$ a sequence $A_{1}, A_{2}, A_{3}, \ldots$ of measurable sets is said to shrink to $x$ nicely if there is a constant $c>0$ for which there is a sequence of positive numbers $r_{1}, r_{2}, r_{3}, \ldots$ with $\lim r_{n}=0$ such that $A_{n} \subset\left(x-r_{n}, x+r_{n}\right)$ and $\lambda\left(A_{n}\right) \geq c \cdot r_{n}$. If a sequence $\left\{A_{n}\right\}_{n}$ shrinks to $x$ nicely and $x$ is a Lebesgue point of $\chi_{F}$, then

$$
d_{F}(x)=\lim _{n \rightarrow \infty} \frac{\lambda\left(F \cap A_{n}\right)}{\lambda\left(A_{n}\right)} .
$$

(See p.140, [13].)

\section{WEAKLY MIXING SKEW PRODUCT ON BERNOULli SHIFT}

Suppose $T$ is a measure-preserving transformation on a probability space $(X, \mu)$. For $\phi: X \rightarrow \mathbb{T}$, if the skew product transformation $T_{\phi}(x, z)=(T x, \phi(x) z)$ on $X \times \mathbb{T}$ is weakly mixing, then we say that $\phi$ is weakly mixing. Let $C(T)$ be the centralizer for $T$, i.e. $C(T)=\{S: S \circ T=T \circ S\}$. We need the following facts.

Lemma 1. Let $T: X \rightarrow X$ be Bernoulli, and let $\phi_{1}, \phi_{2}: X \rightarrow \mathbb{T}$ be weakly mixing. Then there exists $S \in C(T)$ and a measurable function $f: X \rightarrow \mathbb{T}$ such that $\phi_{1} \circ S / \phi_{2}=f \circ T / f$.

For the proof see [14]. In this case we have a commutative diagram:

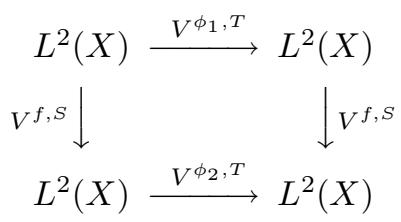

Lemma 2. Let $T: X \rightarrow X$ be Bernoulli, and let $\phi: X \rightarrow \mathbb{T}$ be weakly mixing. Then $T_{\phi}$ is also Bernoulli. Hence $T$ and $T_{\phi}$ are isomorphic, since they are both Bernoulli with the same entropy.

For the proof see [6] and [14]. As a corollary, $V^{\phi, T}: f(x) \mapsto \phi(x) f(T x)$ in $L^{2}(X)$ has absolutely continuous spectrum since $U_{T_{\phi}}$ has countable Lebesgue spectrum on $L^{2}(X \times \mathbb{T})$ and since $V^{\phi, T}$ is spectrally equivalent to $\left.U_{T_{\phi}}\right|_{H_{1}}: H_{1} \rightarrow H_{1}$ where 
$H_{1}=\left\{f(x) \cdot z \in L^{2}(X \times \mathbb{T}): f \in L^{2}(X)\right\}$ and $U_{T_{\phi}}$ is the unitary operator induced by $T_{\phi}$.

Theorem 1. Let $T$ be a Bernoulli transformation on $(X, \mu)$. Define a unitary operator $V^{\phi, T}: f(x) \mapsto \phi(x) f(T x)$ in $L^{2}(X)$. If $\phi$ is weakly mixing, then $V^{\phi, T}$ has countable Lebesgue spectrum.

Proof. By Lemma 1 it suffices to show the existence of weakly mixing $\phi$ such that $V^{\phi, T}$ has Lebesgue spectrum.

First, we replace $T$ on $X$ by $T_{\psi}$ on $X \times \mathbb{T}$ where $\psi: X \rightarrow \mathbb{T}$ is weakly mixing. By Lemma $2, T_{\psi}$ is isomorphic to $T$, so it is enough to prove that $\tilde{T} \equiv T_{\psi}$ has a weakly mixing skew product $\tilde{T}_{\tilde{\phi}}$ such that $V^{\tilde{\phi}, \tilde{T}}$ has countable Lebesgue spectrum where $\tilde{X}=X \times \mathbb{T}$ and $\tilde{\phi}: \tilde{X} \rightarrow \mathbb{T}$.

Put $\tilde{\phi}(\tilde{x})=\tilde{\phi}(x, z)=z$. Then $\left(V^{\tilde{\phi}, \tilde{T}} F\right)(x, z)=z F(\tilde{T}(x, z))$ where $F \in L^{2}(\tilde{X})$. We use the following Fourier type argument: If $f \perp g$, then for any $n \in \mathbb{Z}$,

$$
\begin{aligned}
\left(\left(V^{\tilde{\phi}, \tilde{T}}\right)^{(n)} f, g\right) & =\iint\left(\left(V^{\tilde{\phi}, \tilde{T}}\right)^{(n)} f(x, z)\right) \overline{g(x, z)} d \mu d z \\
& =\iint \tilde{\phi}^{(n)}(x, z) f\left(\tilde{T}^{n}(x, z)\right) \overline{g(x)} d \mu d z \\
& =\iint z^{n} \prod_{i=0}^{n-1} \psi^{(i)}(x) f\left(T^{n} x\right) \overline{g(x)} d \mu d z=0 .
\end{aligned}
$$

We used $\tilde{T}(x, z)=(T x, \psi(x) z)$ and

$$
\begin{aligned}
\tilde{\phi}^{(n)}(x, z) & =\tilde{\phi}(x, z) \tilde{\phi}(\tilde{T}(x, z)) \cdots \tilde{\phi}\left(\tilde{T}^{n-1}(x, z)\right) \\
& =z \cdot \psi(x) z \cdot \psi^{(2)}(x) z \cdots \psi^{(n-1)}(x) z=z^{n} \prod_{i=0}^{n-1} \psi^{(i)}(x) .
\end{aligned}
$$

Now take $f_{n}=f_{n}(x) \in L^{2}(\tilde{X})$ satisfying $f_{n} \perp f_{m}$ if $n \neq m$. Then the cyclic subspaces generated by $f_{n}$ are mutually orthogonal. Similarly we have $\left(\left(V^{\tilde{\phi}, \tilde{T}}\right)^{(n)} f, f\right)$ $=0$ for $n \neq 0$.

\section{A COCYCle Equation FOR ONE-Sided Bernoulli Shift}

First we consider one-sided Bernoulli- $\left(\frac{1}{2}, \frac{1}{2}\right)$ shift, which is measure-theoretically isomorphic to $T: x \mapsto 2 x(\bmod 1)$ on $[0,1)$ with Lebesgue measure $\lambda$. Note that $T^{-1} F \cap[0, r]=\frac{1}{2} F \cap[0, r]$ for $0<r \leq \frac{1}{2}$, and $T^{-1} F \cap[r, 1]=\left(\frac{1}{2} F+\frac{1}{2}\right) \cap[r, 1)$ for $\frac{1}{2} \leq r<1$.

Definition 1. For a fixed set $F$ and a real $0 \leq t<1$, define a continuous function $h_{F, t}(r)$ on $(0,1-t)$ by

$$
h_{F, t}(r) \equiv h_{t}(r)=\frac{\lambda(F \cap[t, t+r])}{r} .
$$

Note that $d_{F}^{+}(t)=\lim _{r \rightarrow 0+} h_{F, t}(r)$.

Lemma 3. If $E \subset\left[\frac{1}{2}, 1\right)$ and $E=F \triangle T^{-1} F$ for some set $F$, then (i) $h_{0}\left(\frac{r}{2^{n}}\right)=$ $h_{0}(r)$ for $n \in \mathbb{N}, 0<r \leq 1$; (ii) if $d_{F}^{+}(0)$ exists, then $d_{F}^{+}(0)=h_{0}(r)=0$ or 1 for every $r$. 
Proof. (i) Take $0<r \leq 1$. Since $\lambda\left(\left(F \triangle T^{-1} F\right) \cap\left[0, \frac{r}{2}\right]\right)=0$, we have $F \cap\left[0, \frac{r}{2}\right]=$ $T^{-1} F \cap\left[0, \frac{r}{2}\right]$. Hence $\lambda\left(F \cap\left[0, \frac{r}{2}\right]\right)=\lambda\left(T^{-1} F \cap\left[0, \frac{r}{2}\right]\right)=\lambda\left(\frac{1}{2} F \cap\left[0, \frac{r}{2}\right]\right)=\frac{1}{2} \lambda(F \cap[0, r])$ and $h_{0}\left(\frac{r}{2}\right)=h_{0}(r)$. Thus $h_{0}\left(\frac{r}{2^{n}}\right)=h_{0}\left(\frac{r}{2^{n-1}}\right)=\cdots=h_{0}(r)$.

(ii) Since $h_{0}\left(\frac{r}{2^{n}}\right)=h_{0}(r)$, for all $n \in \mathbb{N}$ and $0 \leq r<1$ by (i),

$$
d_{F}^{+}(0)=\lim _{s \rightarrow 0+} \frac{\lambda(F \cap[0, s])}{s}=\lim _{n \rightarrow \infty} h_{0}\left(\frac{r}{2^{n}}\right)=h_{0}(r) .
$$

Assume that $d_{F}^{+}(0)=\alpha, 0<\alpha<1$. Since for every $0 \leq r<1$, there exists sufficiently small $\delta(r)>0$ such that $0 \leq r+\epsilon<1$ for all $0<\epsilon<\delta(r)$, i.e.,

$$
\begin{gathered}
\frac{\lambda(F \cap[0, r+\epsilon])}{r+\epsilon}=\alpha, \\
\lambda(F \cap[r, r+\epsilon])=\lambda(F \cap[0, r+\epsilon])-\lambda(F \cap[0, r]) \\
=\alpha(r+\epsilon)-\alpha r=\alpha \epsilon .
\end{gathered}
$$

Hence $\lambda(F \cap[r, r+\epsilon]) / \epsilon=\alpha$, so $r$ has right metric density $\alpha$ for all $0 \leq r<1$. Since $0<\alpha<1$, we arrive at a contradiction.

Hence we investigate the existence of $d_{F}^{+}(0)$ in Lemmas 4 and 5 .

Lemma 4. Let $E=F \triangle T^{-1} F$. If $E \subset\left[\frac{1}{2}, 1\right)$ is a finite union of intervals with dyadic endpoints, then there exists an $r_{0}$ such that for $t=\left[c_{1}, \ldots, c_{l}\right], h_{t}\left(\frac{r}{2^{n}}\right)=h_{t}(r)$ and $h_{t} \equiv h_{0}$ or $h_{t} \equiv 1-h_{0}$ for $n \in \mathbb{N}$ and $0<r \leq \frac{r_{0}}{2^{l}}$.

Proof. Let $E=\bigcup_{i=1}^{m}\left[a_{i}, b_{i}\right]$ with $a_{i}=\left[a_{i, 1}, \ldots, a_{i, p_{i}}\right]$ and $b_{i}=\left[b_{i, 1}, \ldots, b_{i, q_{i}}\right]$ for $i=1, \ldots, m$. Put $r_{0}=\frac{1}{2^{k}}$ where $k=\max \left\{p_{1}, \ldots, p_{m}, q_{1}, \ldots, q_{m}\right\}$.

Step I. We consider the case for $l=1$. By Lemma 3 we have $h_{0}(r)=h_{0}\left(\frac{r}{2^{n}}\right)$ for $0<r \leq r_{0}$ and $\lambda\left(E \cap\left[\frac{1}{2}, \frac{1}{2}+\frac{r_{0}}{2}\right]\right)=0$ or $\lambda\left(E \cap\left[\frac{1}{2}, \frac{1}{2}+\frac{r_{0}}{2}\right]\right)=\frac{r_{0}}{2}$.

Case 1. Assume $\lambda\left(E \cap\left[\frac{1}{2}, \frac{1}{2}+\frac{r_{0}}{2}\right]\right)=0$. Since $E=F \Delta T^{-1} F, \lambda\left(F \cap\left[\frac{1}{2}, \frac{1}{2}+r\right]\right)=$ $\lambda\left(T^{-1} F \cap\left[\frac{1}{2}, \frac{1}{2}+r\right]\right)=\lambda\left(T^{-1} F \cap[0, r]\right)=\frac{1}{2} \lambda(F \cap[0,2 r])$. Thus $h_{\frac{1}{2}}(r)=h_{0}(2 r)=$ $h_{0}(r)$, and $h_{\frac{1}{2}}\left(\frac{r}{2^{n}}\right)=h_{0}\left(\frac{r}{2^{n}}\right)=h_{0}(r)=h_{\frac{1}{2}}(r)$ for $0<r \leq \frac{r_{0}}{2}$.

Case 2. Assume $\lambda\left(E \cap\left[\frac{1}{2}, \frac{1}{2}+\frac{r_{0}}{2}\right]\right)=\frac{r_{0}}{2}$. Then

$$
\begin{aligned}
\lambda\left(F \cap\left[\frac{1}{2}, \frac{1}{2}+r\right]\right) & =r-\lambda\left(T^{-1} F \cap\left[\frac{1}{2}, \frac{1}{2}+r\right]\right) \\
& =r-\lambda\left(T^{-1} F \cap[0, r]\right)=r-\frac{1}{2} \lambda(F \cap[0,2 r]) .
\end{aligned}
$$

Thus $h_{\frac{1}{2}}(r)=1-h_{0}(2 r)=1-h_{0}(r)$ and $h_{\frac{1}{2}}\left(\frac{r}{2^{n}}\right)=1-h_{0}\left(\frac{r}{2^{n}}\right)=1-h_{0}(r)=h_{\frac{1}{2}}(r)$ for $n \in \mathbb{N}$ and $0<r \leq \frac{r_{0}}{2}$. Hence $h_{\frac{1}{2}}\left(\frac{r}{2^{n}}\right)=h_{\frac{1}{2}}(r)=h_{0}(r)$ or $h_{\frac{1}{2}}\left(\frac{r}{2^{n}}\right)=1-h_{0}(r)$.

Step II. By induction we assume that if $s=\left[s_{1}, \ldots, s_{l-1}\right]$, then $h_{s}\left(\frac{r}{2^{n}}\right)=h_{s}(r)$ for all $0<r \leq \frac{r_{0}}{2^{l-1}}$ and $h_{s}=h_{0}$ or $1-h_{0}$.

Let $t=\left[c_{1}, \ldots, c_{l}\right]$ and $s=\left[c_{2}, \ldots, c_{l}\right]$; then $t=\left[0, c_{2}, \ldots, c_{l}\right]$ or $t=\left[1, c_{2}, \ldots, c_{l}\right]$. If $t=\left[0, c_{2}, \ldots, c_{l}\right]$, then $t=\frac{1}{2} s$, and if $t=\left[1, c_{2}, \ldots, c_{l}\right]$, then $t=\frac{1}{2} s+\frac{1}{2}$. Note that $\lambda\left(E \cap\left[t, t+\frac{r_{0}}{2^{l}}\right]\right)=0$ or $\lambda\left(E \cap\left[t, t+\frac{r_{0}}{2^{l}}\right]\right)=\frac{r_{0}}{2^{l}}$.

Case 1. Assume $\lambda\left(E \cap\left[t, t+\frac{r_{0}}{2^{l}}\right]\right)=0$. Then $\lambda(E \cap[t, t+r])=0$ for $0<r \leq \frac{r_{0}}{2^{l}}$. Since $E=F \triangle T^{-1} F$,

$$
\lambda(F \cap[t, t+r])=\lambda\left(T^{-1} F \cap[t, t+r]\right)=\frac{1}{2} \lambda(F \cap[s, s+2 r]) .
$$


Thus

$$
h_{t}(r)=h_{s}(2 r)=h_{s}(r)=h_{0}(r) \quad \text { or } \quad h_{t}(r)=1-h_{0}(r)
$$

and

$$
h_{t}\left(\frac{r}{2^{n}}\right)=h_{s}\left(\frac{r}{2^{n}}\right)=h_{s}(r)=h_{t}(r)
$$

for $0<r \leq \frac{r_{0}}{2^{l}}$.

Case 2. If $\lambda\left(E \cap\left[t, t+\frac{r_{0}}{2^{t}}\right]\right)=\frac{r_{0}}{2^{t}}$, then $\lambda(E \cap[t, t+r])=r$ for $0<r \leq \frac{r_{0}}{2^{l}}$. Since

$$
\begin{aligned}
\lambda(F \cap[t, t+r]) & =r-\lambda\left(T^{-1} F \cap[t, t+r]\right) \\
& =r-\frac{1}{2} \lambda(F \cap[s, s+2 r]),
\end{aligned}
$$

we have

$$
h_{t}(r)=1-h_{s}(2 r)=1-h_{s}(r)=h_{0}(r) \quad \text { or } \quad h_{t}(r)=1-h_{0}(r)
$$

and

$$
h_{t}\left(\frac{r}{2^{n}}\right)=1-h_{s}\left(\frac{r}{2^{n}}\right)=1-h_{s}(r)=h_{t}(r) .
$$

Hence for $t=\left[c_{1}, \ldots, c_{l}\right]$ we have

$$
h_{t}\left(\frac{r}{2^{n}}\right)=h_{t}(r)=h_{0}(r) \quad \text { or } \quad h_{t}\left(\frac{r}{2^{n}}\right)=1-h_{0}(r) .
$$

Remark. By Lemma 4 we know that $d_{F}^{+}(0)$ exists if and only if $d_{F}^{+}(t)$ exists for every dyadic point $t$ and if it exists, then $d_{F}^{+}(t)=d_{F}^{+}(0)$ or $d_{F}^{+}(t)=1-d_{F}^{+}(0)$.

Lemma 5. Let $E=F \triangle T^{-1} F$. If $E \subset\left[\frac{1}{2}, 1\right)$ is a finite union of intervals with dyadic endpoints, then every dyadic point has the right metric density for $F$.

Proof. By Lemma 4 it suffices to show that the point 0 has the right metric density for $F$. Hence we assume that $\lim _{r \rightarrow 0+} h_{0}(r)$ does not exist. Let $r_{0}$ be the same as in Lemma 4. We see that for $t=\left[c_{1}, \ldots, c_{l}\right], h_{t}\left(\frac{r}{2^{n}}\right)=h_{t}(r)=h_{0}(r)$ or $1-h_{0}(r)$ for $0<r \leq \frac{r_{0}}{2^{l}}$ by Lemma 5 .

Take a Lebesgue point $\xi$ of $\chi_{F}$, with $d_{F}(\xi)=1$ and put $r_{n}=\frac{r_{0}}{2^{n}}$. For every $n$ take $\xi_{n}$ in the set of dyadic numbers $\left\{\left[c_{1}, \ldots, c_{m}\right]: m \leq n\right\}$ so that the sequence $\xi_{n}$ converges to $\xi$ and $\left[\xi_{n}, \xi_{n}+r_{n}\right] \subset\left(\xi-\frac{1}{2^{n-1}}, \xi+\frac{1}{2^{n-1}}\right)$. Since

$$
\frac{\lambda\left(\left[\xi_{n}, \xi_{n}+r_{n}\right]\right)}{2^{2-n}}=\frac{r_{0}}{4}
$$

the subsets $\left[\xi_{n}, \xi_{n}+r_{n}\right]$ shrink to $\xi$ nicely. For a fixed $r_{0}$, there exists $\epsilon>0$ such that $\epsilon<h_{0}\left(r_{0}\right)<1-\epsilon$. If not, the metric density at the point 0 exists. Hence for all $n$,

$$
\frac{\lambda\left(F \cap\left[\xi_{n}, \xi_{n}+r_{n}\right]\right)}{r_{n}}=h_{\xi_{n}}\left(r_{n}\right)=h_{0}\left(r_{n}\right)
$$

or

$$
1-h_{0}\left(r_{n}\right)<1-\epsilon
$$

Since $d_{F}(\xi)=1$, we have a contradiction. 
Lemma 6. Let $E=F \triangle T^{-1} F$. If $E$ is a finite union of intervals with dyadic endpoints and there exists a dyadic number $p>0$ such that $E \supset[0, p)$, then there is an $r_{0}$ satisfying the following:

(i) $h_{0}\left(\frac{r}{2^{2 n}}\right)=h_{0}(r)$ and $h_{0}\left(\frac{r}{2^{2 n-1}}\right)=1-h_{0}(r)$ for all $n \in \mathbb{N}$ and $0<r \leq r_{0}$.

(ii) For $t=\left[c_{1}, \ldots, c_{l}\right], h_{t}\left(\frac{r}{2^{2 n}}\right)=h_{t}(r), h_{t}\left(\frac{r}{2^{2 n-1}}\right)=1-h_{t}(r)$ and $h_{t} \equiv h_{0}$ or $h_{t} \equiv 1-h_{0}$ for $n \in \mathbb{N}$ and $0<r \leq \frac{r_{0}}{2^{l}}$.

(iii) There is no measurable set $F$ such that $E=F \triangle T^{-1} F$.

Proof. Let $E=\bigcup_{i=1}^{m}\left[a_{i}, b_{i}\right]$ with $a_{i}=\left[a_{i, 1}, \ldots, a_{i, p_{i}}\right]$ and $b_{i}=\left[b_{i, 1}, \ldots, b_{i, q_{i}}\right]$ for $i=1, \ldots, m$. Put $r_{0}=\frac{1}{2^{k}}$ where $k=\max \left\{p_{1}, \ldots, p_{m}, q_{1}, \ldots, q_{m}\right\}$.

(i) Take $r, 0<r \leq r_{0}$. Since $\lambda\left(\left(F \triangle T^{-1} F\right) \cap\left[0, \frac{r}{2}\right]\right)=\frac{r}{2}$, we have $\lambda\left(F \cap\left[0, \frac{r}{2}\right]\right)=$ $\frac{r}{2}-\lambda\left(T^{-1} F \cap\left[0, \frac{r}{2}\right]\right)=\frac{r}{2}-\lambda\left(\frac{1}{2} F \cap\left[0, \frac{r}{2}\right]\right)=\frac{r}{2}-\frac{1}{2} \lambda(F \cap[0, r])$ and $h_{0}\left(\frac{r}{2}\right)=1-h_{0}(r)$. Hence $h_{0}\left(\frac{r}{2^{2 n}}\right)=1-h_{0}\left(\frac{r}{2^{2 n-1}}\right)=\cdots=h_{0}(r)$ and $h_{0}\left(\frac{r}{2^{2 n-1}}\right)=1-h_{0}\left(\frac{r}{2^{2 n-2}}\right)=\cdots=$ $1-h_{0}(r)$.

(ii) By (i), $h_{0}\left(\frac{r}{2^{2 n}}\right)=h_{0}(r)$ and $h_{0}\left(\frac{r}{2^{2 n-1}}\right)=1-h_{0}(r)$ for $n \in \mathbb{N}$ and $0<r \leq r_{0}$, and $\lambda\left(E \cap\left[\frac{1}{2}, \frac{1}{2}+\frac{r_{0}}{2}\right]\right)=0$ or $\lambda\left(E \cap\left[\frac{1}{2}, \frac{1}{2}+\frac{r_{0}}{2}\right]\right)=\frac{r_{0}}{2}$. Now proceed as in Lemma 4 .

(iii) Take $\xi, r_{0}, r_{n}$ and $\xi_{n}$ as in Lemma 5 . Then

$$
d_{F}(\xi)=\lim _{n \rightarrow \infty} h_{\xi_{n}}\left(r_{n}\right)=1-h_{0}\left(r_{0}\right) \text { or } h_{0}\left(r_{0}\right)
$$

by the relations (i, ii). If $d_{F}(\xi)=1-h_{0}\left(r_{0}\right)$, then $h_{0}\left(r_{0}\right)=0$ and $E \neq F \triangle T^{-1} F$ for this $F$.

Remark. For $T: x \mapsto k x(\bmod 1)$, we can use the same argument: Put $E=$ $\bigcup_{i=1}^{m}\left[a_{i}, b_{i}\right]$ with $k$-adic endpoints $\frac{1}{k} \leq a_{i}<b_{i} \leq 1$ for $i=1, \ldots, m$. If $E=F \Delta$ $T^{-1} F$ for some set $F$, then

(i) $h_{0}\left(\frac{r}{k^{n}}\right)=h_{0}(r)$ for all $n \in \mathbb{N}$ and for all $0<r \leq 1$.

(ii) If $d_{F}^{+}(0)$ exists, then $d_{F}^{+}(0)=h_{0}(r)=0$ or 1 .

The existence of $d_{F}^{+}(0)$ is proved similarly as in Lemmas 4 and 5 . As in Lemma 6 , if there is a $k$-adic number $p>0$ such that $E \supset[0, p)$, then there is no measurable set $F$ such that $E=F \triangle T^{-1} F$.

Proposition 1. Let $T$ be defined by $T: x \mapsto k x(\bmod 1)$ on $[0,1)$ and let $E$ be a finite union of intervals with $k$-adic endpoints. If there exists an integer $l, 0 \leq l<k$, such that $E^{c} \supset\left[\frac{l}{k}, \frac{l+1}{k}\right]$, then $\pm \exp \left(\pi i \chi_{E}\right)$ is not a coboundary.

Proof. For the sake of notational simplicity, we consider the case $k=2$. The other case is proved by exactly the same argument.

Assume $E \subset\left[\frac{1}{2}, 1\right]$ and $\exp \left(\pi i \chi_{E}\right)$ is a coboundary. Then by Lemmas 4 and 5 $d_{F}^{+}(0)$ exists, and $d_{F}^{+}(0)=0$ or 1 by Lemma 3 . If $d_{F}^{+}(0)=1$, then $h_{0}(1)=d_{F}^{+}(0)=1$, and

$$
h_{0}(1)=\lambda(F \cap[0,1])=1 .
$$

Thus $F=X$. If $d_{F}^{+}(0)=0$, then $h_{0}(1)=d_{F}^{+}(0)=0$, and

$$
h_{0}(1)=\lambda(F \cap[0,1])=0 .
$$

Thus $F^{c}=X$. So there is no measurable subset $F$ such that $E=F \triangle T^{-1} F$. Since $-\exp \left(\pi i \chi_{E}\right)=\exp \left(\pi i \chi_{E^{c}}\right)$ and $E^{c} \supset\left[0, \frac{1}{2}\right),-\exp \left(\pi i \chi_{E}\right)$ is not a coboundary.

Assume that $E \subset\left[0, \frac{1}{2}\right]$. We know that $T$ is measure-theoretically isomorphic to itself by $\psi: x \mapsto x+\frac{1}{2}(\bmod 1)$. Since $\psi(E) \subset\left[\frac{1}{2}, 1\right]$ and coboundaries are sent to coboundaries by an isomorphism, the conclusion follows. 
Corollary 1. For the transformation $T$ defined by $x \mapsto k x(\bmod 1)$ for $k \geq 2$, a real-valued function $f(x)$ of modulus one is not a coboundary if the discontinuities $0<x_{1}<\cdots<x_{n} \leq 1$ of $f(x)$ are $k$-adic points and $x_{1} \geq \frac{1}{k}$.

Proof. Note that there exists a measurable set $E$ such that $f(x)= \pm \exp \left(\pi i \chi_{E}\right)$ and $E$ is a finite union of $k$-adic endpoints with $E^{c} \supset\left[0, \frac{1}{k}\right]$.

\section{A COCYCle EQUATION FOR TWO-SIDED BeRnoulli ShifT}

Let $(Y, \mathcal{C}, \mu)$ be a probability space, $f \in L^{1}(Y, \mathcal{C}, \mu)$ and $\mathcal{B} \subset \mathcal{C}$ a sub- $\sigma$-algebra. Define a complex measure $\nu(B)=\int_{B} f d \mu, B \in \mathcal{B}$. By the Radon-Nikodym Theorem, there is a function $g \in L^{1}(Y, \mathcal{B}, \mu)$ such that $\nu(B)=\int_{B} g d \mu$. We use the notation $E(f \mid \mathcal{B})$ for $g$, and call it the conditional expectation of $f$ with respect to $\mathcal{B}$. Also let $S$ be a transformation defined on $Y$. Then we say that $\mathcal{B}$ is an exhaustive $\sigma$-algebra if $S^{-1} \mathcal{B} \subset \mathcal{B}$ and $S^{n} \mathcal{B} \uparrow \mathcal{C}$. The Martingale Theorem states that $E\left(f \mid S^{n} \mathcal{B}\right)$ converges to $f$ a.e. and in $L^{1}(Y, \mathcal{C}, \mu)$ for $f \in L^{1}(Y, \mathcal{C}, \mu)$.

Proposition 2. Let $S$ be a transformation on $(Y, \mathcal{C}, \mu)$, let $\mathcal{B}$ be an exhaustive $\sigma$ algebra $\mathcal{B} \subset \mathcal{C}$, and let $f: Y \rightarrow \mathbb{T}$ be a $\mathcal{B}$-measurable map to the circle group $\mathbb{T}$. If $q: Y \rightarrow \mathbb{T}$ is a $\mathcal{C}$-measurable solution to the equation $f \cdot q=q \circ S$, then $q$ is $\mathcal{B}$-measurable.

Proof. We follow the idea in [11]. Applying the conditional expectation operator $E(\cdot \mid \mathcal{B})$ to the equation

$$
f \cdot q=q \circ S
$$

then $f \cdot E(q \mid \mathcal{B})=E(q \circ S \mid \mathcal{B})$ or $f \cdot E(q \mid \mathcal{B})=E(q \mid S \mathcal{B}) \circ S$. Multiplying this with $(*)$ inverted we have

$$
\overline{q(y)} \cdot E(q \mid \mathcal{B})(y)=\overline{q(S y)} \cdot E(q \mid S \mathcal{B}) \circ S(y) \quad \text { a.e. }
$$

so that

$$
\int_{Y} \bar{q} \cdot E(q \mid \mathcal{B}) d \mu=\int_{Y} \bar{q} \cdot E(q \mid S \mathcal{B}) d \mu .
$$

By exactly the same argument, using $S^{n} \mathcal{B}$ in place of $\mathcal{B}$, we have

so that

$$
\int_{Y} \bar{q} \cdot E\left(q \mid S^{n} \mathcal{B}\right) d \mu=\int_{Y} \bar{q} \cdot E\left(q \mid S^{n+1} \mathcal{B}\right) d \mu
$$

$$
\int_{Y} \bar{q} \cdot E(q \mid \mathcal{B}) d \mu=\int_{Y} \bar{q} \cdot E\left(q \mid S^{n} \mathcal{B}\right) d \mu .
$$

Taking limits, we get $\int_{Y} \bar{q} \cdot E(q \mid \mathcal{B}) d \mu=\int_{Y}|q|^{2} d \mu$.

Thus $E(q \mid \mathcal{B})=q$ a.e., and $q$ is $\mathcal{B}$-measurable.

Definition 2. Let $k$ be fixed and $B=\bigcup_{i=1}^{m} B_{i}$ where

$$
B_{i}=\left\{y \mid\left(y_{0}, \ldots, y_{n_{i}}\right)=\left(a_{i, 0}, \ldots, a_{i, n_{i}}\right)\right\} .
$$

We call $B$ an elementary set generated by $B_{1}, \ldots, B_{m}$. If there exists an integer $l$, $0 \leq l<k$, such that $a_{i, 0} \neq l$ for all $0 \leq i \leq m$, then we say that $B_{1}, \ldots, B_{m}$ have the same missing initial digit.

Theorem 2. For the two-sided Bernoulli- $\left(\frac{1}{k}, \ldots, \frac{1}{k}\right)$ shift with $k \geq 2$, a function $\pm \exp \left(\pi i \chi_{B}\right)$ is not a coboundary if $B$ is an elementary set generated by a finite union of blocks with the same missing initial digit. 
Proof. Without loss of generality, we assume that $B$ is a cylinder set generated by a block. Let $S$ be a shift on the Bernoulli- $\left(\frac{1}{k}, \ldots, \frac{1}{k}\right)$ shift space $(Y, \mathcal{C}, \mu)$. Let $B=\left\{y \mid\left(y_{0}, \ldots, y_{n}\right)=\left(a_{0}, \ldots, a_{n}\right)\right\}$ be a cylinder set generated by a block $\left(a_{0}, \ldots, a_{n}\right)$ of finite length.

Let $\mathcal{B} \subset \mathcal{C}$ be a sub- $\sigma$-algebra generated by the cylinder sets

$$
B=\left\{y \mid\left(y_{0}, \ldots, y_{m}\right)=\left(b_{0}, \ldots, b_{m}\right)\right\} .
$$

Then $\mathcal{B}$ is an exhaustive $\sigma$-algebra of $\mathcal{C}$. If $f \cdot q=q \circ S$, then $q$ is $\mathcal{B}$-measurable by Proposition 2. Since $\left(Y, \mathcal{B}, \mu_{\mid \mathcal{B}}, S\right)$ is measure-theoretically isomorphic to $(X, \mathcal{A}, \lambda, T)$ where $X=[0,1), \mathcal{A}$ is a Borel $\sigma$-algebra, $\lambda$ is Lebesgue measure, and $T$ is defined by $x \mapsto k x(\bmod 1)$, it follows that $f(y)$ is a coboundary if and only if $\exp \left(\pi i \chi_{[a, b]}(x)\right)$ is a coboundary where $a=\left[a_{0}, \ldots, a_{n}\right], b=\left[a_{0}, \ldots, a_{n}+1\right]$. Hence $f(y)$ is not a coboundary by Proposition 1 .

For the finite union of blocks with the same missing initial digit, the proof is similar.

\section{ACKNOWLEDGEMENT}

The authors thank M. Lemańczyk for helpful discussions.

\section{REFERENCES}

[1] Y. Ahn and G. H. Choe, On normal numbers mod 2, Colloq. Math. 76 (1998), 161-170. CMP 98:11

[2] G. H. Choe, Spectral types of uniform distribution, Proc. Amer. Math. Soc. 120 (1994), 715-722. MR 94e:47014 MR 94e:47014

[3] _ Ergodicity and irrational rotations, Proc. R. Ir. Acad. 93A (1993), 193-202. MR 94m:28017

[4] _ Products of operators with singular continuous spectra, Proc. Sympos. Pure Math., 51, Part 2 (1990), 65-68. MR 91h:47006

[5] J. P. Conze, Remarques sur les transformations cylindriques et les equations fonctionnelles, Séminaire de Probabilité I, Rennes, France (1976). MR 58:28427

[6] J. Feldman, D. J. Rudolph and C. C. Moore, Affine extensions of a Bernoulli shift, Trans. Amer. Math. Soc. 257 (1980), 171-191. MR 81a:28014

[7] H. Helson and W. Parry, Cocycles and spectra, Ark. Mat. 16 (1978), 195-206. MR 80d:28036

[8] A. Iwanik, M. Lemańczyk and D. Rudolph, Absolutely continuous cocycles over irrational rotations, Israel J. Math. 83 (1993), 73-95. MR 94i:58108

[9] R. B. Kirk, Sets which split families of measurable sets, Amer. Math. Monthly 79 (1972), 884-886. MR 47:5201

[10] H. A. Medina, Spectral types of unitary operators arising from irrational rotations on the circle, Michigan. Math. J. 41(1) (1994), 39-49. MR 95a:28014

[11] W. Parry, A Cocycle equation for shift, Contemp. Math. 135 (1992), 327-333. MR 93j:28028

[12] K. Petersen, Ergodic Theory, Cambridge Univ. Press London, 1983. MR 87i:28002

[13] W. Rudin, Real and Complex Analysis, Third edition, McGraw-Hill, 1987. MR 88k:00002

[14] D. J. Rudolph, Classifying the isometric extensions of a Bernoulli shift, J. Analyse Math. 34 (1978), 36-60. MR 80g:28020

[15] P. Walters, An Introduction to Ergodic Theory, Springer-Verlag New York, 1982. MR 84e:28017

Korea Advanced Institute of Science and Technology, Kusong-dong, Yusong-gu, 305-701 TAEJON, Korea

E-mail address: ahntau@math.kaist.ac.kr

E-mail address: choe@euclid.kaist.ac.kr 